\title{
Frontières
}

\section{Les auditions de la Commission spéciale sur la question de mourir dans la dignité dans trois villes du Québec}

\section{Éric Volant}

Volume 24, numéro 1-2, automne 2011, printemps 2012

L’aide médicale à mourir

URI : https://id.erudit.org/iderudit/1013094ar

DOI : https://doi.org/10.7202/1013094ar

Aller au sommaire du numéro

Éditeur(s)

Université du Québec à Montréal

ISSN

1916-0976 (numérique)

Découvrir la revue

Citer cet article

Volant, É. (2011). Les auditions de la Commission spéciale sur la question de mourir dans la dignité dans trois villes du Québec. Frontières, 24(1-2), 113-117. https://doi.org/10.7202/1013094ar d'utilisation que vous pouvez consulter en ligne. 


\section{Les auditions \\ de la Commission spéciale sur la question de mourir dans la dignité dans trois villes du Québec}

\author{
Éric Volant, Ph. D., \\ professeur associé, \\ Département de sciences des religions, UQAM.
}

Aux fins d'analyse des débats lors des auditions publiques de la Commission spéciale de l'Assemblée nationale du Québec sur la question de mourir dans la dignité, nous avons choisi dans le Journal des débats sept séances ${ }^{1}$ tenues à Trois-Rivières, à Sherbrooke et à Québec. Nous avons analysé les transcriptions de ces sessions. Cet échantillonnage nous a permis de recueillir, de classifier et d'examiner des matériaux assez pertinents pour nous permettre d'identifier certaines lignes majeures de l'opinion publique exprimée durant ces sessions. Ce premier déblayage pourra contribuer ultérieurement à un examen plus global de l'ensemble des transcriptions mises à la disposition $\mathrm{du}$ public, des mémoires déposés et de la compilation des réponses au questionnaire en ligne ${ }^{2}$.

\section{MOURIR DANS LA DIGNITÉ ET AUTONOMIE}

La confusion dans la définition des termes est un problème récurrent tout au long des débats, parfois même dans les propos de certains membres de la Commission. Il en va ainsi de la notion de dignité, même si celle-ci est clairement définie dans le document de présentation des objectifs de la Commission.
Un premier constat, c'est celui de l'étroite association que les participants établissent entre «la dignité humaine et l'autonomie du sujet» $(41,5)$. La notion de dignité s'y trouve très liée au droit inaliénable du sujet à choisir sa manière de vivre et de mourir.

Un des membres de l'Association québécoise de défense des droits des retraités et des préretraités (AQDR), section TroisRivières, s'exprime ainsi: "la notion de dignité devant la vie et la mort est subjective et [...], par conséquent, il appartient aux personnes en fin de vie, et seulement à elles, de déterminer ce que signifie mourir dans la dignité et choisir la manière avec laquelle elles entendent de vivre leur fin de vie.» $(41,5)$

Une personne, souffrant d'une «maladie neurogénétique incurable et dégénérative » et bénévole à un centre de prévention du suicide, estime que "mourir dans la dignité» est pour elle un exercice de libre choix, car son estime de soi lui refuse d'être "à la charge de personne ", ni d'être «un fardeau pour sa famille» $(41,5)$. Elle «ne pense pas rendre service à la société que d'être couchée, légume, dans un lit, sans bouger. [Elle] permet peut-être aux autres d'être bons [envers elle], peut-être, mais ce n'est peut-être pas le témoignage qu' [elle veut] faire dans sa vie» $(41,5)$. C'est sa fierté, sa peur de la honte et son estime de soi qui sont en jeu.
Une dame en chaise roulante apporte son témoignage: "c'est moins une préoccupation avec la question de mourir dans la dignité que de savoir comment est-ce que je fais pour vivre dans la dignité, et j'associe beaucoup la question de dignité avec le maintien de mon autonomie, ma capacité d'agir, mon "empowerment", qui, pour moi, se conjugue sur trois plans. Le premier, c'est, il faut que je sois capable, que j'aie une capacité de choisir, d'avoir des options devant moi et d'avoir cette possibilité d'effectuer un choix parmi les différentes options. On enlève l'option et on enlève une partie de la dignité » $(41,5)$.

Pour la plupart de ceux qui s'expriment en faveur de l'euthanasie, la dignité de la personne, interprétée en association étroite avec l'autonomie, est perçue comme un droit au libre choix en lien avec son pouvoir de maîtriser la vie et la mort. Elle se traduit, selon la malade et bénévole citée ci-dessus, en termes du droit à la propriété: «La mort m'appartient, ma mort m'appartient» $(41,5)$, sous-entendue, la libre disposition de son corps ou de sa vie. «La vie n'appartient pas à celui qui veille près du lit, mais elle appartient à celui qui est dans le lit et qui souffre» $(42,21)$. Afin de promouvoir le libre choix, on cite volontiers des philosophes ou des experts en éthique: «Il me semble qu'on fait bon marché de l'autonomie et du respect de la personne ainsi que de la notion des 
droits individuels ( $\mathrm{P}^{\mathrm{r}}$ Kluge)» $(41,18)$; cela demeure «une minorité qui peut et veut pouvoir choisir librement le moment de mourir (Tillich)» $(41,18)$, mais il s'agit d'une minorité non négligeable qui «mérite d'être exaucée $\left(\mathrm{D}^{\mathrm{r}} \mathrm{E}\right.$. Cassell) $)(41,18)$, car «ce n'est pas la vie qui est sacrée, c'est la personne» (É. Fuchs) $(41,5)$. Ainsi, une demande d'euthanasie "faite en toute lucidité d'esprit et de liberté ne doit pas être considérée comme un privilège, mais comme un droit fondamental de liberté de choix et d'autonomie» $(41,5)$. "Le droit de choisir sa destinée jusqu'à son terme en accord avec ses valeurs, ses croyances est une question de liberté humaine, d'autonomie et de droit individuel» $(41,5)$. «La liberté de choisir de son sort» doit tenir compte «des responsabilités qu'elle a à l'égard de la collectivité ». Celles-ci pourraient se résumer en trois points: le premier veut dire «respecter la dignité humaine»; le deuxième, "favoriser une décision sereine et éclairée » et le troisième : "une décision morale implique des droits, mais aussi des responsabilités» $(41,21)$.

Cette équivalence entre dignité humaine et choix autonome en fin de vie est contestée par un participant au débat, mais celuici réduit l'autonomie explicitement à la capacité physique, celle de la performance ou de la productivité. Celle-ci, étant pour une bonne part absente chez les aînés, les handicapés et les personnes en fin de vie, ne les empêche pas pour autant d'exercer leurs facultés intellectuelles en fonction de leur discernement moral. Cette autonomie responsable est soumise à l'épreuve en temps de maladie et de souffrance où fragilisée, elle devra pourtant pouvoir encore s'affirmer et s'exercer. On note que cette capacité de libre choix du mourant en tant que personne autonome est souvent mise en doute dans le milieu des soins palliatifs et on déplore une certaine condescendance, qui fait peur à plusieurs des futurs patients qui craignent d'être réduits, dans la relation médecin-patient, à un statut de bénéficiaires des soins accordés avec bienveillance. Un médecin affirme: «je trouve important pour moi de promouvoir un respect malgré le fait de sa dépendance, malgré le fait de sa maladie, malgré toutes les conséquences qui s'en suivent, de la maladie» $(41,24)$. Il est vrai que le malade n'est pas indigne parce qu'il est dépendant de soins; la souffrance physique ou morale n'affecte pas nécessairement sa liberté d'esprit et de décision. «Est-il possible alors de vivre dignement jusqu'au bout?», se demande un participant. Il répond: «Les soins palliatifs mettent tout en œuvre pour préserver la relation à travers laquelle s'exprime la confiance. Être solidaire face à la souffrance de l'autre exige alors de la soulager avec tous les moyens que la science nous permet de soigner avec dignité » $(41,18)$.

\section{LE TRAITEMENT DE LA SOUFFRANCE}

$\mathrm{Au}$ sujet du soulagement ou de la suppression de la souffrance, le clivage entre ceux qui favorisent les soins palliatifs et ceux qui optent, à certaines conditions, pour l'euthanasie est très prononcé. Or, c'est au niveau même de la perception ou de la compréhension de la souffrance que la différence se manifeste en premier lieu. Ce désaccord se révèle déjà dans l'approche cognitive de la souffrance.

Ainsi, en soins palliatifs, on situe l'«impasse » au plan méthodologique : il y a absence de définition consensuelle «de ce qu'est la douleur intolérable» $(41,28)$. Cet agnosticisme d'ordre scientifique «invalide toute démarche dans le sens d'une légalisation des pratiques » dites euthanasiques $(41,28)$. "L'homme n'est pas détruit par la souffrance, il est détruit par la souffrance qui n'a aucun sens.» Afin de faire face à la souffrance, il doit, à même sa vulnérabilité, apprendre à mieux connaître les potentialités de son être et même à «se surpasser». Par ailleurs, au niveau de la pratique, il ne serait pas juste de prétendre que le processus $\mathrm{du}$ mourir est «souvent sinon toujours marqué par des souffrances importantes et incoercibles» $(41,24)$. En effet, «les recherches prouvent que, jusqu'à 40\% des cancéreux [...] n'expérimenteront pas de douleur [à entendre: «physique»] durant la trajectoire de la dernière maladie». Et «lorsqu'ils sont pris en charge par une équipe expérimentée de soins palliatifs », les souffrances sont « rarissimes» $(41,24)$.

Dans le discours des soins palliatifs, la souffrance, dite morale ou psychique, semble être perçue et traitée uniquement en tant que dépression: « la dépression est donc très souvent à l'origine d'une souffrance pouvant conduire à la demande de l'euthanasie. Du point de vue médical, soigner la dépression par la suppression $\mathrm{du}$ malade ne peut absolument pas constituer un traitement approprié même si «une dépression clinique justifie, aux Pays-Bas, l'acte euthanasique » selon la sociologue Céline Fontaine, citée par le $\mathrm{D}^{\mathrm{r}}$ Serge Daneault $(41,24)$, car la souffrance peut être soulagée par la médication et l'accompagnement. À la question : «Que fait-on pour les personnes non dépressives qui sont pleinement conscientes de leur fin, mais qui, en même temps, ne trouvent pas réponse à travers toutes les avancées médicales des dernières années?» $(41,24)$, on poursuit l'argumentation comme si toute souffrance était, par principe ou par essence, dépressive: «Ce n'est pas facile de dépister une dépression, [...] puis de faire un bon traitement, parce que ça demande un accompagnement... il y a des réussites, il y a des échecs» $(41,24)$. On va jusqu'à découvrir dans la prétendue popularité de l'euthanasie un signe d'une "dépression collective», marquée par la fragilité des sujets, particulièrement les jeunes et les vieillards.

Or, «tous les experts reconnaissent que certaines souffrances demeurent inapaisables ». L'AQDR de Trois-Rivières cite sur ce point le $\mathrm{D}^{\mathrm{r}}$ Marcel Boisvert. Ce dernier se réfère à Éric Cassell, le père de la notion de souffrance, qui estime que "prétendre que toute souffrance peut être soulagée dénote une ignorance de la vraie nature de la souffrance» $(41,5)$. C'est d'ailleurs parmi les personnes lourdement handicapées, participant aux débats, et les proches témoins de personnes gravement malades ainsi que chez certains médecins, que l'on entend un autre son de cloche que le discours empreint de dolorisme qui met en valeur la souffrance en tant que contribution au bien de la société: "Pourquoi la souffrance d'autrui aideraitelle à faire avancer la société ?» $(41,5)$. Il y a des souffrances insupportables et insensées qui ne peuvent être soulagées. $\mathrm{Au}$ sujet des soins palliatifs dans les hôpitaux du Québec, on entend même parler de torture: "quand ça vient aux douleurs et aux souffrances psychologiques en logeant l'usager en chambre partagée avec un usager également à la fin de sa vie, nous torturons» $(41,19)$; «[...] l'agonie d'un autre, la douleur et les pleurs des visiteurs d'un autre et finalement le décès d'un autre, la terreur que votre tour est proche, la restriction d'accès aux personnes aimées » $(41,19)$ deviennent insoutenables. "La douleur de savoir que la vie n'est qu'une question de jours domine tout. Le seul remède [...] l'amour: la présence et l'intimité des gens aimés. Un remède impossible en chambre partagée. L'absence de ce remède est pure torture» $(41,19)$. Le droit à une chambre individuelle en soins palliatifs ou à des soins à domicile doit être mieux respecté. Et dans les hôpitaux, il y a un besoin criant d'un plus grand nombre de lits.

En soins palliatifs, la sédation palliative est interprétée comme "légale» et justifiée du point de vue éthique en tant qu'option à double effet. Or cela paraît manifester quelque «proximité » avec l'euthanasie, mais «la philosophie et l'approche» sont différentes, même si l'effet, c'est-à-dire la mort, est le même. Par contre, «tout comme les médecins québécois, ceux de Belgique et des Pays-Bas demeurent perplexes quant aux frontières entre sédation et euthanasie 
et même quant à l'intentionnalité de l'euthanasie ou de l'option du double effet, car la réalité veut que, lorsqu'une sédation terminale est amorcée, aucun médecin ne souhaite qu'elle se prolonge indûment, ce qui revient à désirer qu'elle soit courte, donc à espérer une mort prochaine, à espérer une mort qu'on ne veut pas causer, tout en posant des gestes qui peuvent la précipiter: la proximité de ces frontières éthiques les rend indéchiffrables, d'où le réalisme humaniste de la notion de soins appropriés mise de l'avant par le Collège des médecins» $(41,18)$. C'est le $\mathrm{D}^{\mathrm{r}}$ Marcel Boisvert qui s'exprime ainsi et qui y ajoute une critique sévère: "Voilà un mourant qu'on ne peut soulager, rendu à une semaine d'une sédation terminale qui le mènera à son décès dans l'inconscience, à qui l'on impose ce scénario. Je ne sais comment concevoir une mort sereine dans ces circonstances. Je crains que je n'aie d'autre qualificatif qu'acharnement palliatif » $(41,18)$.

Selon d'autres participants qui se sont prononcés, la sédation terminale est «un accompagnement pour aider quelqu'un à quitter, d'une façon inconsciente » et "l'euthanasie, c'est aider quelqu'un à mourir consciemment: le mourant dit: bye, bye, je m'en vais» $(41,5)$. Les médecins qui sont ouverts à l'euthanasie «sont plus ouverts à l'écoute du patient qu'à leur agenda personnel». Ils ne sont pas pour autant «moins humains» et accordent la sédation terminale pour soulager les mourants qui le désirent. Par ailleurs, le refus des longues sédations, observé chez plusieurs patients, est signe que ce traitement est insupportable pour celui qui le subit, mais aussi «intolérable pour les proches et stressant pour les soignants » $(41,5)$.

\section{LES SOINS PALLIATIFS}

Le $\mathrm{D}^{\mathrm{r}}$ Serge Daneault, médecin en soins palliatifs, fait les trois recommandations suivantes: la création d'unités de soins palliatifs dans tous les établissements de 600 décès et plus par année, la mise en place d'équipes de consultation en soins palliatifs dans les petits hôpitaux en région, la formation d'équipes en soins palliatifs et un financement séparé des soins palliatifs à domicile $(41,24)$. Il propose «un changement de culture médicale » et «l'élargissement des soins palliatifs à d'autres clientèles que le cancer» $(41,24)$. Cependant, il s'oppose à «la création de maisons de soins palliatifs partout au Québec», car il ne veut pas que ces maisons deviennent le «modèle québécois », favorisant ainsi une forme de «privatisation de la mort» $(41,24)$. Pour lui, il s'agit de «la protection des plus vul- nérables de la société» $(41,24)$. Advenant la légalisation de l'euthanasie, des préalables s'imposeraient que nous pourrions résumer ainsi : informer la population de son droit de refuser un traitement et de son droit de recevoir des soins palliatifs et un accompagnement; mettre à jour les connaissances des médecins en traitement des douleurs et d'autres symptômes; recommander aux médecins l'utilisation des opiacés; voir au financement adéquat et optimal des soins palliatifs, prodigués par des professionnels formés, dans un environnement approprié $(41,24)$.

Les soins à domicile sont le souhait de la majorité des Québécois. Il faut en faire une priorité. C'est préférable à une chambre partagée dans les hôpitaux et aux centres de soins palliatifs en région. Par contre, le contrôle de la douleur est plus difficile à la maison. Il faudrait offrir une formation adéquate aux bénévoles et aux proches à la maison. «Mourir dans l'intimité» plutôt que «mourir dans la dignité!» $(41,5)$. Cependant, selon le $\mathrm{D}^{\mathrm{r}}$ Boisvert, qui a été membre d'une équipe de soins palliatifs, on entend que les soins palliatifs ont été institués et ont progressé "dans une mentalité religieuse avouée $[\ldots]$; les soins palliatifs ont très nettement attiré des gens très religieux [...]; la pierre d'achoppement des soins palliatifs, c'est d'abréger la vie, l'euthanasie, hein? Alors, les soins palliatifs sont incapables d'intérioriser la notion même d'abréger la vie même juste un peu» $(41,18)$. Un homme laisse entendre ses réticences à l'égard de certains comportements, trop maternels et infantilisants: "Ma femme sait que je ne suis pas le genre à être cajolé, à me faire jouer des chansons, à me faire flatter » $(41,5)$. À l'opposé, la D ${ }^{\text {re }}$ Michèle Dallaire, médecin en soins palliatifs, raconte: «il y a une femme encore l'autre semaine qui disait : Écoutez, moi, une chance que j'ai eu la chance de venir ici, parce que je suis en train de vivre les plus beaux moments de ma vie dans votre unité de soins palliatifs, parce qu'elle, être dorlotée comme ça, elle n'avait jamais vu ça. C'est une femme qui était isolée et le milieu comme ça, prendre soin comme ça, c'était une nouvelle vie pour elle. Elle ne voulait pas mourir, mais pas du tout, je dois vous dire, elle voulait que ça dure le plus longtemps possible. En général, c'est ça que les gens veulent en soins palliatifs, c'est vivre le plus longtemps possible» $(41,18)$.

Les soins palliatifs jouissent, en principe, de la faveur générale. Même ceux que l'on appelle les «pro-euthanasies»se prononcent en faveur des soins palliatifs et ne recommandent la légalisation de l'euthanasie que pour une minorité dont les souffrances physiques et morales ne sont pas «apaisables», autrement dit: ne peuvent être soulagées.

\section{L'EUTHANASIE ET LE SUICIDE ASSISTÉ}

Une dame, opposée à l'euthanasie, se pose la question: "Pourquoi ne pas mettre à leur disposition un accès au suicide par dose létale qu'ils prendront au moment où ils l'auront décidé, en compagnie de leur famille ou [d'une personne aidante] s'ils ne peuvent le faire eux-mêmes? Voilà la différence entre l'euthanasie, qui est une mort décidée par d'autres ou un meurtre par compassion, et le suicide, soit par dose létale qu'on prend soi-même ou assisté quand on ne peut le faire seul » $(41,24)$. Au cours de l'interrogation, elle insiste cependant sur la nécessité du testament biologique ou d'un mandat d'inaptitude afin de pouvoir exprimer ses dernières volontés et semble donc s'opposer uniquement à l'euthanasie non volontaire ou non désirée, c'est-à-dire imposée par le médecin ou la famille. Une autre participante pense que «le suicide assisté, c'est uniquement dans les cas de personnes qui ne sont pas capables de le faire elles-mêmes. Quand je parle de suicide autonome, je dirais, là, ça serait une dose létale que la personne décide de prendre au moment que ça lui convient, parce qu'on parle toujours de souffrance physique. Maintenant, l'euthanasie, dans mon livre à moi, c'est une décision qu'une personne prend envers une autre. Si c'est à sa demande, bien, reconnaissons... soyons politically rigides, puisque c'est une demande de suicide. Puis je ne vois pas pourquoi le médecin dirait: Tiens, je t'accorde ou je... moi, je vais te tuer parce que tu me le demandes. Non. Fais-le toi-même quand tu le jugeras nécessaire parce tu es capable, toujours dans les souffrances physiques» $(41,24)$.

Il $\mathrm{y}$ a une distinction à faire entre les deux interventions: une décision d'un malade, jeune ou vieux, en fin de vie dont les souffrances physiques ou psychiques sont devenues insupportables, et la décision d'un jeune ou d'un aîné ne se trouvant pas dans une situation de fin de vie, mais qui, pour des raisons d'ordre mental, moral ou psychique, décide de s'enlever la vie. Son geste ne sera pas pénalisé, car il est soustrait à la loi. Mais il est surtout à retenir que l'euthanasie est définie dans nos sociétés comme un acte accompli par le médecin à la demande de la personne gravement malade en fin de vie, tandis que le suicide assisté est un geste posé par la personne elle-même avec l'assistance du médecin, dont le rôle ne consiste qu'à lui procurer les produits pharmaceutiques appropriés. 
La prévention du suicide demeure un travail de première importance dans les cas du suicide des jeunes et des aînés hors des situations de maladie grave et de fin de vie. Mais elle ne fait pas partie de l'objet de la présente Commission. Cependant, on ne peut pas légitimement prouver l'existence de liens de cause à effet entre la légalisation de l'euthanasie et du suicide assisté en fin de vie, d'une part, et le suicide des jeunes et des aînés ou de toutes les autres personnes vulnérables ou fragilisées, d'autre part. La légalisation de l'euthanasie peut exercer un «effet d'entraînement», d'imitation ou de contagion sur un plan strictement personnel, comme un participant l'exprime en termes simples: "si l'euthanasie est acceptée par ma grand-mère, pourquoi moi, jeune, je ne puis pas me libérer de ma souffrance par le suicide?» $(41,24)$.

\section{LA LÉGALISATION DE L'EUTHANASIE ET DU SUICIDE ASSISTÉ}

Selon le Barreau du Québec, « le rôle du droit n'est pas de départager les partisans et les adversaires de l'euthanasie et du suicide assisté; le Barreau croit qu'il faille respecter de façon absolue les idées et les valeurs de chacun. Le respect de la volonté de la personne en fin de vie nous apparaît être la meilleure et la plus fiable balise qui assurerait un espace de liberté suffisant de façon à permettre à chacun de donner un sens à un moment essentiel de la vie, et ce, selon ses propres valeurs» $(41,8)$.

La légalisation de l'euthanasie se situe dans une certaine logique de l'évolution du droit en regard du respect de la liberté de la personne. On évoque qu'«au Canada le suicide et la tentative de suicide ne sont pas interdits depuis 1972 » et «depuis 1994, toute personne peut refuser des traitements médicaux même si cette décision aura comme conséquence la mort du patient [...] Soyons donc conséquents et respectons cette même logique de la volonté de la personne qui, en phase terminale, demande l'aide de mourir» $(41,5)$. Il demeure, cependant, dans la pratique qu'il n'est pas toujours évident dans quelle mesure une personne fait un libre choix. Un médecin belge aurait cité le cas d'un patient de 80 ans qui avait demandé l'euthanasie, mais il s'avéra que ce n'était pas lui qui voulait l'euthanasie, «c'est son fils qui, finalement, écrit la lettre qu'il fait signer à son père, et qui donne lieu à une euthanasie, alors, qui raccourcit la période de vie de cette personne-là, qui était malade, de... de mois, peut-être même d'une année ou deux» $(41,24)$. Il importe d'étudier chaque cas individuellement. Par ailleurs, l'expérience nous apprend qu'il ne faut pas sous-estimer la liberté avec laquelle une personne de 85, 90 ans et plus fait son choix, même si elle est gravement malade ou mourante.

L'euthanasie, même utilisée de façon rarissime, devrait avoir une place parmi les formes d'accompagnement d'un patient en fin de vie, si "l'on se soucie vraiment des besoins véritables, spécifiques et singuliers de chaque mourant» $(41,5)$. Il en va du «droit de choisir sa destinée jusqu'à son terme en accord avec ses valeurs, ses croyances », selon " des critères et des exigences rigoureux». Les personnes qui font "un choix éclairé de mourir» estiment "qu'en fin de vie la qualité est préférable à la quantité. Pour eux l'important n'est pas d'ajouter des années à la vie, mais plutôt d'ajouter de la vie aux années» $(41,5)$.

Somme toute, l'euthanasie est "une stratégie qui a pour finalité de mettre rapidement un terme à sa vie » $(41,5)$, là où l'approche des soins palliatifs en est une «qui vise d'abord à soulager les souffrances physiques, morales, psychologiques intolérables» $(41,5)$. Les deux stratégies produisent cependant le même effet, celui de la mort. La seconde, utilisant le protocole de la sédation terminale, se cache derrière l'argument de la bonne intention, tandis que la première annonce ouvertement l'effet visé. Or, il est contreindiqué d'opposer les soins palliatifs à l'euthanasie, car celle-ci «se situe dans la toute fin » des soins palliatifs. Un médecin ne voit «absolument pas pourquoi ses collègues disent que les soins palliatifs vont disparaître» $(41,5)$, si on légalise l'euthanasie. Les soins palliatifs et l'euthanasie peuvent faire partie de la même pratique médicale. D'où «l'importance de la formation des autres intervenants dans le milieu de la santé » $(41,5)$ à l'ensemble d'un processus global de traitement de la douleur et d'accompagnement en fin de vie. Il est opportun de «créer un cadre légal où le législateur, tout en protégeant les personnes fragilisées par la maladie [...] et en protégeant également le corps médical, puisse permette à toute personne humaine atteinte d'une maladie incurable en phase terminale de demander et d'obtenir de l'aide à mourir» $(41,5)$.

Cependant, d'autres jugent que l'on ne doit pas légiférer pour une minorité, mais «pour une majorité, oui!», comme si les minorités n'avaient pas de droit à une législation qui les inclut en répondant à leurs besoins spécifiques. "Dans la vie, on n'a pas toujours ce que l'on veut», dit quelqu'un, comme si les personnes gravement souffrantes, qui demandent l'euthanasie, étaient du genre capricieux. Plus apocalyptique encore: "si on légalisait l'euthanasie, on mettrait en péril toute la mission intrinsèque de la médecine et en péril le réseau de la santé», comme si la mission de la médecine et le régime québécois de la santé ne pouvaient s'ouvrir à des perceptions, à des soucis, à des besoins ou à des situations du monde contemporain. "Si on légifère, on s'en va vers une banalisation de la vie humaine» $(41,19)$, au sens qu'on lui enlève son «caractère sacré et intouchable».

On se plaint d'une valorisation excessive des soins palliatifs lorsque ses adeptes font «de la survie biologique un absolu ». Le jésuite médecin Marc Desmet a fait une mise en garde contre le danger «d'absolutiser la survie biologique». Des philosophes et moralistes sont d'accord avec l'idée que «la personne avec un $\mathrm{P}$ majuscule a préséance sur la vie avec un $\mathrm{V}$ majuscule, même à partir de Pie XII » (41, $18)$. On «est loin de nier la grande valeur de la vie, mais elle ne lui accorde pas un caractère sacré intouchable», comme le soutient l'AQDR, section Trois-Rivières. Nous remarquons que les positions dans ce débat dépendent des perceptions que les participants, les uns et les autres, ont développées face aux rapports entre la vie et la mort: "Si la mort fait partie intégrante de la vie, alors la mort est une étape de la vie. Le droit de mourir avec dignité devrait être protégé comme n'importe quel autre aspect du droit à la vie (M. Cory)» $(41,5)$.

Autre point important, c'est la relation thérapeutique, la relation du médecin et de l'équipe médicale avec le malade. Dans le passé, disent des médecins, «nous nous sommes souciés du bien du patient, de le soulager et de l'accompagner». Maintenant, ils s'interrogent: "sommes nous prêts à respecter sa volonté, à lui reconnaître le droit de décider lui-même de son bien? Avant, c'étaient les médecins qui décidaient, là on essaie de collaborer avec le patient ». L'un deux se permet de citer un texte paru dans l'Encyclopédie sur la mort et soumis à la Commission : «Il importe de situer la question d'aide à mourir à l'intérieur d'un modèle particulier de relation thérapeutique. Le malade et le médecin se considèrent avec leurs compétences respectives, comme des partenaires égaux, libres et autonomes, engagés dans une communication bilatérale et dans une responsabilité partagée. Le malade se trouve, selon son expérience de la maladie et de la souffrance, au centre du processus décisionnel comme un interlocuteur valable et légitime» $(41,5)$. Le $\mathrm{D}^{\mathrm{r}}$ Boisvert définit l'autonomie comme un pouvoir partagé: «Le pouvoir est toujours partagé par deux personnes. Le jésuite médecin Marc Desmet a parlé de l'autonomie-enlien avec deux traits d'union, un nom composé, l'euthanasie en lien, une perle. L'autonomie, qui revient à reconnaître 
l'autonomie du patient, donc c'est le médecin qui doit juger de la justification du geste, mais juste le patient est capable de dire si c'est un geste bienfaisant oui ou non» $(41,18)$.

Madame V. Hivon, vice-présidente de la Commission, pense qu'en fin de vie "c'est fondamental de tisser des liens de confiance et une continuité parce qu'elle peut faire des demandes changeantes, difficiles, donc établir cette relation-là » $(41,19)$. Ce lien interdépendant a été bien ressenti par le député $\mathrm{D}^{\mathrm{r}} \mathrm{A}$. Khadir: "Comme médecin, comment je peux être sûr que mon jugement est supérieur au jugement de la personne? Comment je peux estimer que mon jugement est supérieur comme médecin à sa place? Qu'est-ce que je possède pour dire ça?» $(41,24)$. Une dame du groupe «Vive la vie» lui répond: "vous connaissez la maladie mieux que lui, je m'imagine ». Elle craint que la décision prise par un malade créera un précédent. «Si l'on permet à une seule personne de s'enlever la vie ou si on l'assiste à enlever sa vie [...], pourquoi pas moi, pourquoi pas vous, pourquoi pas ma voisine? » $(41,24)$. C'est ainsi que l'on entre dans le vif d'un sujet que l'on désigne par plusieurs noms: la pente glissante, la dérive ou le dérapage.

\section{LE RISQUE DE DÉRIVE}

Une crainte, exprimée dans les débats à plusieurs reprises, est celle des pressions économiques, institutionnelles, sociales et même familiales qui s'exercent sur les aînés et les plus vulnérables de la société. Devenus «fardeau social», ces groupes pourraient constituer des boucs émissaires ou des victimes que l'on sacrifierait injustement pour des raisons utilitaristes. Ce risque à portée aussi grave qu'antihumaniste devra être prévenu dans la formulation même de toute législation concernant l'euthanasie et le suicide assisté.

Ce qui frappe dans les débats, c'est l'espace qui y était occupé par des jugements portés sur les Pays-Bas et la Belgique où l'euthanasie a été légalisée. Certains commentaires sont favorables: «force est d'admettre qu'il n'y a pas dans les pays où les États ayant légalisé l'euthanasie et le suicide assisté, d'abus à ces égards. Ce sont des actes de compassion» $(41,18)$. Un médecin affirme: "Moi, j’ai rencontré personnellement des médecins belges, français, hollandais qui ont pratiqué de l'euthanasie, puis je vous jure, s'ils s'étaient ici, sur le plan de l'humanisme, sur le plan de la compassion, sur le plan de la compétence, ils n'ont rien à nous envier » $(41,18)$.

D'autres appréciations, cependant, sont non seulement défavorables, mais négatives au point d'être inexactes en s'appuyant sur des chiffres non vérifiés et d'être partisanes et subjectives. La peur de la pente glissante est exploitée à excès et déforme les données recueillies. Voyons quelques-unes de ces statistiques: "en 2009, on a 2636 décès par euthanasie, soit $45 \%$ de plus qu'en 2003, $15 \%$ de plus qu'en 2008. Ce chiffre-là ne compte pas les 550 euthanasies involontaires, sans consentement, les 400 cas de suicide assisté ni les 20\% d'euthanasies qui, de toute façon, ne sont pas rapportées comme le stipule la loi. Deux études récentes, fin 2010, révèlent qu'en Flandres [...] une euthanasie sur deux n'est pas rapportée au comité d'évaluation de contrôle fédéral, une sur deux. Et 32\% des euthanasies qui sont réalisées en Flandres le sont sans consentement» $(41,28)$.

Le $\mathrm{D}^{\mathrm{r}}$ Marcel Boisvert tente de rétablir les faits et de rectifier les chiffres du rapport McGill qui «cite à l'indicatif présent une donnée périmée qui devrait l'être à l'imparfait. Il cite la page $30 \mathrm{du}$ rapport où on lit: « $50 \%$ des cas néerlandais d'aide médicale au suicide ou à l'euthanasie ne sont pas rapportés ». Or les deux sources sont de 1992 et 2004 et se réfèrent à des faits antérieurs à 1990 et à 2001, année des révisions officielles néerlandaises : van der Maas et collaborateurs. Or, un rapport publié dans le New England Journal de 2007 très souvent cité résume les données de 1990, 1995, 2001 et 2005. Les pourcentages de cas rapportés pour ces années sont respectivement de 18\%, 40\%, $54 \%$ et $80 \%$ en 2005 . D'un coup d'œil, on voit là une quasi-ligne droite dont la pente raide pointe au-delà de $95 \%$ en 2010 , c'està-dire un maximum atteignable dans ce genre de compilations. Nous sommes très loin de $50 \%$, qui remonte à des données antérieures à $2001 »(41,18)$.

Selon des médecins québécois, qui ont consulté leurs collègues aux Pays-Bas et en Belgique, ceux-ci leur ont recommandé de «ne pas légaliser l'euthanasie, mais ils refusent, sauf une exception, en même temps d'être publiquement identifiés, phénomène qui pose une question en lui-même. Certains de ces médecins pratiquent parfois l'euthanasie et ils vivent alors beaucoup de difficultés et de troubles personnels. Plusieurs d'entre eux avouent avoir subi la pression, l'intimidation de la part des patients et des familles désireuses d'obtenir l'euthanasie» $(41,24)$.

Aux Pays-Bas, où l'euthanasie des moins de 16 ans n'est pas légale, les médecins ont défini le "protocole de Groningen selon lequel il est pratiquées annuellement autour de 20 euthanasies chez des enfants qui ne donnent pas leurs consentements » $(41,24)$. Ce protocole exige pourtant le consentement éclairé des jeunes concernés.
«Comment expliquer qu'aux Pays-Bas, se demande le $\mathrm{D}^{\mathrm{r}}$ Daneault, en plus de 30 ans de pratique euthanasique et en dépit d'un apparent système sophistiqué de recours et de surveillance, il n'y ait eu aucune poursuite pénale à la suite de dizaines de milliers d'euthanasies?» $(41,24)$.

Tous ces chiffres sont-ils exacts? La description offerte par des médecins québécois qui prônent les soins palliatifs correspond-elle à la réalité ? Selon des membres du Barreau du Québec, «si on sait qu'il y a des dérapages ailleurs, comme en Hollande, par exemple, ou en Belgique, bien, c'est parce qu'il $\mathrm{y}$ a des règles. Pas de règle, on ne peut pas établir qu'il $\mathrm{y} a$ un dérapage. Alors, ça prend des règles pour dire: Bien, voici. Là, on est capable d'établir: Un tel s'y conforme, un tel ne s'y conforme pas» $(41,8)$.

\section{UN DÉBAT À SUIVRE}

Les thèmes relevés ici à partir des propos exprimés lors de sept séances de la Commission spéciale de l'Assemblée nationale du Québec sur la question de mourir dans la dignité pourront être enrichis en tenant compte des propos exprimés tout au long des vingt-neuf jours d'audition. Les consultations se sont terminées le 22 mars 2011.

Le lien entre la dignité et l'autonomie, les positions contrastées sur le traitement de la souffrance, la place qui sera faite aux pratiques d'euthanasie et d'aide au suicide soit dans le continuum des soins palliatifs, soit en position d'incompatibilité avec eux - ainsi que le risque de dérive feront partie des thèmes susceptibles de polariser le nouveau débat qui va maintenant s'amorcer à la suite de la publication, en mars 2012, des recommandations formulées par la Commission.

\section{Notes}

1. Les extraits analysés sont tirés des séances suivantes: Trois-Rivières, le 24 septembre 2010 , vol. $41, \mathrm{n}^{\circ} 5$. Sherbrooke, le 25 novembre 2010 , vol. $41, \mathrm{n}^{\circ} 18$; le 26 novembre 2010 , vol 41 , $\mathrm{n}^{\circ}$ 19. Québec, le 2 novembre 2010, vol. 41, $n^{\circ} 15$; le 30 septembre 2010 , vol. $41, n^{\circ} 8$; le 9 février 2011, vol. 41, n 24 et le 17 février 2011, vol. $41, n^{\circ} 28$. Les autres villes visitées par la commission étaient: Montréal, Gatineau, SaintJérôme, Rimouski, et Saguenay.

2. Lors des 29 jours d'auditions publiques, 239 personnes et organismes ont été entendus; 114 personnes ont aussi été entendues pendant les périodes de micro ouvert, alors que les citoyens présents dans la salle d'auditions pouvaient exposer leurs points de vue pendant quelques minutes. Avant la période d'auditions publiques, la Commission avait entendu 32 experts pendant 6 jours. Elle a reçu 273 mémoires et 6558 répondants ont rempli le questionnaire en ligne proposé sur le site Internet de l'Assemblée nationale. 